Census of Ireland. Factors associated with self-harm and repetition of self-harm were compared between the homeless and those living at fixed residence using multivariable-adjusted logistic regression models.

Results There were 58,747 presentations of self-harm in total, of which $3.9 \%$ were among the homeless. The ASIR of selfharm was 30 times higher among the homeless (5,572 presentations per 100,000) compared with those living at fixed residence (187 presentations per 100,000). Intentional overdose was the most common method of self-harm for all; relative to those with fixed residence, homeless individuals were significantly more likely to use minor tranquillisers (47\% vs $39 \%$, $\left.\chi^{2}: p<0.001\right)$ or street drugs $\left(19 \%\right.$ vs. $\left.6 \%, \chi^{2}: p<0.001\right)$ for overdose. Homeless people had significantly higher odds of self-harm repetition within 12 months (vs. fixed residence, AOR 1.46, 95\%CI 1.21-1.77). Within the homeless population, the odds of self-harm repetition were significantly increased among those who engaged in self-cutting (vs. overdose, AOR 1.76, 95\%CI 1.17-2.65) and those who did not receive psychiatric review at index presentation (vs. reviewed, AOR 1.54, 95\%CI 1.05-2.26).

Conclusion There is a disproportionate burden of self-harm among the homeless. Those who present with self-cutting, and who do not receive psychiatric review at index presentation, are particularly vulnerable to repetition. Although this study only reflects self-harm presenting to hospital, and assumes no change in homelessness status during follow-up, it is the largest study of self-harm among the homeless conducted to date. The results may be used to strengthen suicide prevention efforts through earlier recognition of high-risk homeless individuals.

\section{P65 HYPERTENSION IN PREGNANCY PREVALENCE, RISK FACTORS AND OUTCOMES FOR WOMEN BIRTHING IN IRELAND}

${ }^{1} \mathrm{~L}$ Corrigan*, ${ }^{2} \mathrm{~A}$ O'Farrell, ${ }^{1} \mathrm{D}$ Daly, ${ }^{1} \mathrm{P}$ Moran, ${ }^{2} \mathrm{~V}$ Delaney. ${ }^{1}$ Nursing and Midwifery, Trinity College Dublin, Dublin, Ireland; ${ }^{2}$ Health Intelligence Unit, Health Service Executive, Dublin, Ireland

\subsection{6/jech-2019-SSMabstracts.216}

Background Hypertensive disorders of pregnancy account for nearly $18 \%$ of all maternal deaths world-wide. They are associated with increased risk of maternal and neonatal morbidity, adverse pregnancy outcomes and long-term health risks for both mother and baby. This study aims to determine prevalence, risk factors and outcomes associated with hypertensive disorders of pregnancy (Pregnancy-induced hypertension, preeclampsia, eclampsia, HELLP, hypertensive disorder of pregnancy unspecified).

Methods Data on maternity hospital discharges (ICD codes O00-O99) for women giving birth in Ireland in 2016 were extracted from Hospital In-Patient Enquiry (HIPE) using Health Atlas. Women discharged following delivery were identified using ICD-10 codes O80-O84 for delivery. Those with a diagnosis of a hypertensive disorder of pregnancy were identified using ICD-10 codes O10-O16. Frequencies and descriptive statistics were used to present prevalence rates of hypertensive disorders of pregnancy. Pearson's Chi-square and multivariate analyses were conducted to identify risk factors. Data was analysed in SPSS version 25 and JMP version 9.

Results Of 60,188 births, 3531 women (5.9\%) had a hypertensive disorder of pregnancy. Rates were higher among women with pre-existing diabetes, gestational diabetes, obesity and those age $\geq 40$ years $(\mathrm{p}<0.001)$. Women with a diagnosis of a hypertensive disorder of pregnancy had a higher risk of poor foetal growth (OR 2.6), preterm labour and birth (OR 3.7), placental abruption (OR 2.0), long labour (OR 1.4), instrument-assisted delivery (OR 1.2), caesarean section (OR 1.8 ), postpartum haemorrhage (OR 1.6) and length of stay $\geq 6$ days (OR 5.6).

After adjusting for all factors, obesity (OR 4.3) pre-existing diabetes (OR 3.5), gestational diabetes (OR 1.5) and being aged $\geq 40$ years (1.5) remained significantly associated with being diagnosed with a hypertensive disorder.

Conclusion As the number of overweight and obese pregnant women, women giving birth aged $\geq 40$ years, and those with pre-existing diabetes mellitus or new onset gestational diabetes increase rates of hypertensive disorders of pregnancy will likely become more common obstetric risks. They therefore represent a significant public health concern. These findings can help inform strategies for identifying factors associated with hypertensive disorders of pregnancy.

\section{P66 TAKING NUDGE DIGITAL WITH FOOD CHOICE AT WORK: FROM EVALUATION TO PRACTICAL APPLICATION IN EVERYDAY WORKPLACE SETTINGS}

S Fitzgerald*, F Geaney, IJ Perry. School of Public Health, University College Cork, Cork, Ireland

\subsection{6/jech-2019-SSMabstracts.217}

Background Evidence on effective workplace dietary interventions is limited. The Food Choice at Work trial assessed the comparative effectiveness of a workplace dietary intervention involving nutrition education and system-level dietary modification both alone and in combination versus a control workplace on employees' dietary intakes, nutrition knowledge and health status. An economic evaluation assessed the cost-effectiveness of the interventions from the perspective of healthcare providers in terms of QALYs and employers in terms of monetary benefits (reduced absenteeism).

Methods Four manufacturing workplaces in Ireland were allocated to control, nutrition education (Education), system-level dietary modification (System-level) and nutrition education and system-level dietary modification (Combined). Nutrition education included group presentations, individual consultations and detailed nutrition information. System-level dietary modification included menu modification, fruit price discounts, strategic positioning of healthier alternatives and portion size control. Data on dietary intakes, nutrition knowledge, health status, QALYs and absenteeism were obtained at baseline and at 7-9 months follow-up. Multivariate analysis of covariance compared changes across the groups. The economic evaluation included cost-utility and cost-benefit analyses.

Results Follow-up data were obtained for 541 employees (1864 years) (64\% of 850 recruited). There were significant positive changes in intakes of saturated fat $(p=0.013)$, salt $(p=0.010)$ and nutrition knowledge $(p=0.034)$ between baseline and follow-up in the combined intervention versus the control. Significant changes in BMI $(-1.2 \mathrm{~kg} / \mathrm{m} 2 \quad(\mathrm{p}=0.047)$ were also observed in the combined intervention. System-level modification yielded the highest additional QALYs (€ 101.37/ QALY) and annual net benefit for employers (€ 56.56/ employee). 
Discussion Combining nutrition education and system-level dietary modification is an effective approach for promoting healthy eating at work. The FCW intervention is a sustainable cost-effective model and wide-scale implementation is underway at local, national and international workplaces. Specific elements will be digitally automated to increase the reach of the FCW intervention. The purpose of our next related study is to develop, implement and assess the effectiveness of digitalising the FCW intervention. The results of this study will determine the effectiveness and user engagement of a theorybased, multidimensional, web platform and smartphone nutrition intervention to support healthier food choices and better health status for employees in their workplaces.

\section{P67 DEVELOPMENT OF A DATA QUALITY FRAMEWORK FOR HEALTH AND SOCIAL CARE - A STRATEGIC APPROACH TO ASSESS AND IMPROVE THE QUALITY OF HEALTH DATA AND INFORMATION IN IRELAND}

A Healy, C Duggan, B Foley, R Flynn, T Huss*. Health Information and Standards Directorate, Health Information and Quality Authority (HIQA), City Gate, Mahon, Cork, Ireland

\subsection{6/jech-2019-SSMabstracts.218}

Background A considerable amount of data is collected on a regular basis about health and social care services in Ireland. We rely on this for the planning and delivery of safe and efficient services and to inform future policy and service planning. Therefore, it is vital that there is confidence and trust in the quality of this data in order to support the provision of safe and efficient care to patients and service users. The aim of this research was to develop guidance on the development of a data quality framework for health and social care organisations in Ireland, to enable them to systematically assess, monitor, evaluate and improve the quality of their data and information.

Methods This research involved four key stages; an international review of evidence to compare and contrast international approaches as well as the key components of a data quality framework, the establishment of an Expert Working Group to provide advice and knowledge, interviews and consultation with national and international experts, and finally, the conduct of a targeted consultation with a wide range of stakeholders including national evidence and policy makers, representatives from national data collections, service providers and academia. The findings were collated and assessed thematically in order to inform the finalised guidance.

Results Guidance on a data quality framework for health and social care organisations in Ireland was developed. The components of the framework include: a data quality strategy which outlines key components including audit, governance and training; a data quality assessment tool which provides a detailed set of criteria organisations can use to comprehensively assess its data sources across all five dimensions of quality; reporting on data quality using key performance indicators and data quality statements, and a data quality improvement cycle detailing the continuous approach organisations can undertake to improve the quality of their data and information.

Conclusion The guidance, which is the first of its kind in Ireland, provides tools to support health and social care organisations in ensuring that their data is 'fit for purpose'. By applying this guidance, it is possible for organisations to establish a baseline for data quality and identify areas for improvement. Where organisations adopt a systematic approach to assessing, improving and maintaining the quality of their data, sector-wide improvements in the quality of data can be observed and stakeholders can be assured that the data they use to support decision making is of high quality.

\section{P68 DIVERGENT ASSOCIATIONS BETWEEN LIFE COURSE COGNITIVE TRAJECTORIES AND BRAIN PATHOLOGIES: FINDINGS FROM THE 1946 BRITISH BIRTH COHORT}

\begin{abstract}
${ }^{1,2}{ }^{2} \mathrm{~N}$ James ${ }^{*},{ }^{2} \mathrm{CA}$ Lane, ${ }^{2} \mathrm{TD}$ Parker, ${ }^{2} \mathrm{~K}$ Lu, ${ }^{2} \mathrm{~A}$ Keshavan, ${ }^{2} \mathrm{SM}$ Buchanan, ${ }^{2} \mathrm{SE}$ Keuss, ${ }^{2} \mathrm{DM}$ Cash, ${ }^{2} \mathrm{BB}$ Malone, ${ }^{3} \mathrm{JM}$ Nicholas, ${ }^{2} \mathrm{H}$ Murray-Smith, ${ }^{1} \mathrm{~A}$ Wong, ${ }^{2} \mathrm{NC}$ Fox, ${ }^{2} \mathrm{JM}$ Schott, ${ }^{1} \mathrm{M}$ Richards. ${ }^{1} \mathrm{MRC}$ Unit for Lifelong Health and Ageing at UCL, University College London, London, UK; ${ }^{2}$ Dementia Research Centre, University College London, London, UKi ${ }^{3}$ Department of Medical Statistics, London School of Hygiene and Tropical Medicine, London, UK
\end{abstract}

\subsection{6/jech-2019-SSMabstracts.219}

Background Cognitive function may serve as an early indicator of prodromal Alzheimer's disease (AD). We examined how cognitive measures over the life course are associated with the $\mathrm{AD}$-related biomarkers of amyloid $(\mathrm{A} \beta)$ status and whole brain volume in a population-based sample free of dementia and other major neurological disorders.

Methods Data were from 458 (49\% female) dementia-free participants from Insight 46, a sub-study of the National Survey of Health and Development (1946 British birth cohort). At age 70-72, participants underwent 18F-Florbetapir amyloidPET and multi-modal MRI imaging. Regression analyses and multilevel modelling examined patterns between cognitive measures (spanning age 8-69) and the neuroimaging outcomes of $\mathrm{A} \beta$ status $( \pm)$ and whole brain volume.

Results Of a range of cognitive tests, word-list learning (WLT) at age 69 was specifically associated with $\mathrm{A} \beta$ status at the $5 \%$ level (OR $0.75(0.57,0.96)) . \mathrm{A} \beta+$ individuals additionally showed faster WLT decline in the 26 years preceding imaging (M1: $\beta=-0.06(-0.09,-0.01))$. Measures of reaction time (M1: $\mathrm{b}=-0.17(95 \%$ CI $-0.27,-0.08)$ and search speed (M1: $\mathrm{b}=0.12$ $(95 \%$ CI $0.03,0.22))$ at age 60 , and decline in search speed over 26 years (M1: $\beta=0.01(0.00,0.01))$, were associated with smaller whole brain volume. These patterns remained similar after adjusting for childhood cognition, sex, education, child and adult SEP, affective problems and concurrent pathology. APOE-e4 status attenuated the association between verbal memory and $A \beta$.

Conclusion Associations between cognitive function and neuropathology at age 70-72 appear to manifest between ages 6069 in a population-based sample without dementia or other major neurological problems. In particular, decline in WLT is associated with $\mathrm{A} \beta+$ and is partially attributed to the effects of APOE- $\epsilon 4$; whereas level of reaction time and rate of decline of search speed are associated with smaller brain volume and are APOE- $\epsilon 4$-independent. Our findings are consistent with evidence of cognitive changes as part of an $\mathrm{AD}$ prodromal syndrome in early older-age; and provide evidence that cognitive domains can differentiate underlying pathophysiology associated with $\mathrm{AD}$. 